\title{
Giant pituitary macroadenoma of stem cell origin: illustrative case
}

\author{
David T. Asuzu, MD, PhD, MPH, ${ }^{1,4}$ Rebecca M. Burke, MD, PhD, ${ }^{1}$ Jeffrey Hakim, MD, PhD, ${ }^{1}$ Dylan Coss, MD, ${ }^{2}$ Min S. Park, MD, ${ }^{1}$ \\ Spencer C. Payne, MD, ${ }^{3}$ and John A. Jane Jr., MD ${ }^{1}$
}

${ }^{1}$ Department of Neurosurgery, ${ }^{2}$ Division of Neuropathology, and ${ }^{3}$ Department of Otolaryngology, Head and Neck Surgery, University of Virginia, Charlottesville, Virginia; and ${ }^{4}$ Surgical Neurology Branch, National Institute of Neurological Disorders and Stroke, National Institutes of Health, Bethesda, Maryland

\begin{abstract}
BACKGROUND Giant pituitary macroadenomas with a diameter $>4 \mathrm{~cm}$ are rare tumors, accounting for only about $5 \%$ of pituitary adenomas. They are more difficult to maximally resect safely owing to limited access as well as encasement of adjacent structures. Acidophil stem cell adenomas are rare immature neoplasms proposed to derive from common progenitor cells of somatotroph and lactotroph cells. These adenomas comprise about $4.3 \%$ of surgically removed pituitary adenomas. No previous reports have described acidophil stem cell adenomas that grow to the size of giant macroadenomas. This rare entity poses special challenges given the need for maximal safe resection in an immature neoplasm.

OBSERVATIONS The authors report a 21 -year-old female who presented with 3 years of progressive visual decline and a giant macroadenoma. She underwent endoscopic transsphenoidal surgery for decompression. Given the tumor size and involvement of adjacent critical structures, gross-total resection was not achieved. The authors review the literature on giant pituitary adenomas and provide a discussion on clinical management for this rare entity.
\end{abstract}

LESSONS The authors present a very rare case of a giant pituitary adenoma of acidophil stem cell origin and discuss the technical and management challenges in this rare entity.

https://thejns.org/doi/abs/10.3171/CASE2122

KEYWORDS pituitary adenoma; visual dysfunction; giant macroadenoma; stem cells; transsphenoidal resection

Pituitary adenomas occur in about $14 \%-22 \%$ of patients based on autopsy and radiological studies. ${ }^{1}$ Pituitary adenomas make up about $8 \%$ of central nervous system tumors and $15 \%$ of primary intracranial tumors. ${ }^{2,3}$ Giant pituitary macroadenomas with a diameter $>4 \mathrm{~cm}$ are rare tumors, accounting for only about $5 \%$ of pituitary adenomas. ${ }^{4-6}$ Giant adenomas may require staged or simultaneous transcranial approaches. ${ }^{7}$ The average extent of resection for giant macroadenomas is about $72.8 \%,{ }^{8}$ and lower resection rates are encountered with cavernous sinus invasion. ${ }^{5}$

Acidophil stem cell adenomas comprise about $4.3 \%$ of surgically removed pituitary adenomas. ${ }^{9}$ They are characterized on electron microscopy as unicellular tumors with immature cytoplasm and frequent mitochondrial abnormalities and have a more aggressive clinical profile than well-differentiated adenomas. ${ }^{9}$ No previous reports have described acidophil stem cell adenomas that present morphologically as giant macroadenomas.

\section{Illustrative Case}

A 21-year-old right-handed female presented in 2017 with headaches and progressive visual decline in the left eye. She was evaluated by a primary care physician and was diagnosed with a brain mass. She gradually developed right-sided visual field deficits. She presented to our center in 2020. Ophthalmological examination demonstrated acuity at $1 / 200$ in the right eye and no light perception in the left eye. Visual field testing showed a right temporal visual field defect with relatively preserved central acuity nasally and superior-temporally. Optical coherence tomography (OCT) showed dramatic nerve fiber layer dropouts bilaterally with loss of ganglion cells. Detailed endocrine evaluation revealed no abnormalities. She had no stigmata of Cushing's disease or acromegaly.

Magnetic resonance imaging (MRI) of the brain showed a large enhancing sellar/suprasellar mass measuring approximately $6.4 \times$ $6.1 \times 7 \mathrm{~cm}$ with prominent vascular flow voids (Fig. $1 \mathrm{~A}-\mathrm{C})$. Computed

ABBREVIATIONS ACA = anterior cerebral artery; $\mathrm{CTA}=$ computed tomography angiography; $\mathrm{DWI}=$ diffusion weighted imaging; $\mathrm{ICA}=$ internal carotid artery; $\mathrm{MCA}=$ middle cerebral artery; MRI = magnetic resonance imaging; OCT = optical coherence tomography.

INCLUDE WHEN CITING Published Month day, 2021; DOI: 10.3171/CASE2122.

SUBMITTED January 10, 2021. ACCEPTED January 13, 2021.

(c) 2021 The authors, CC BY-NC-ND 4.0 (http://creativecommons.org/licenses/by-nc-nd/4.0/). 


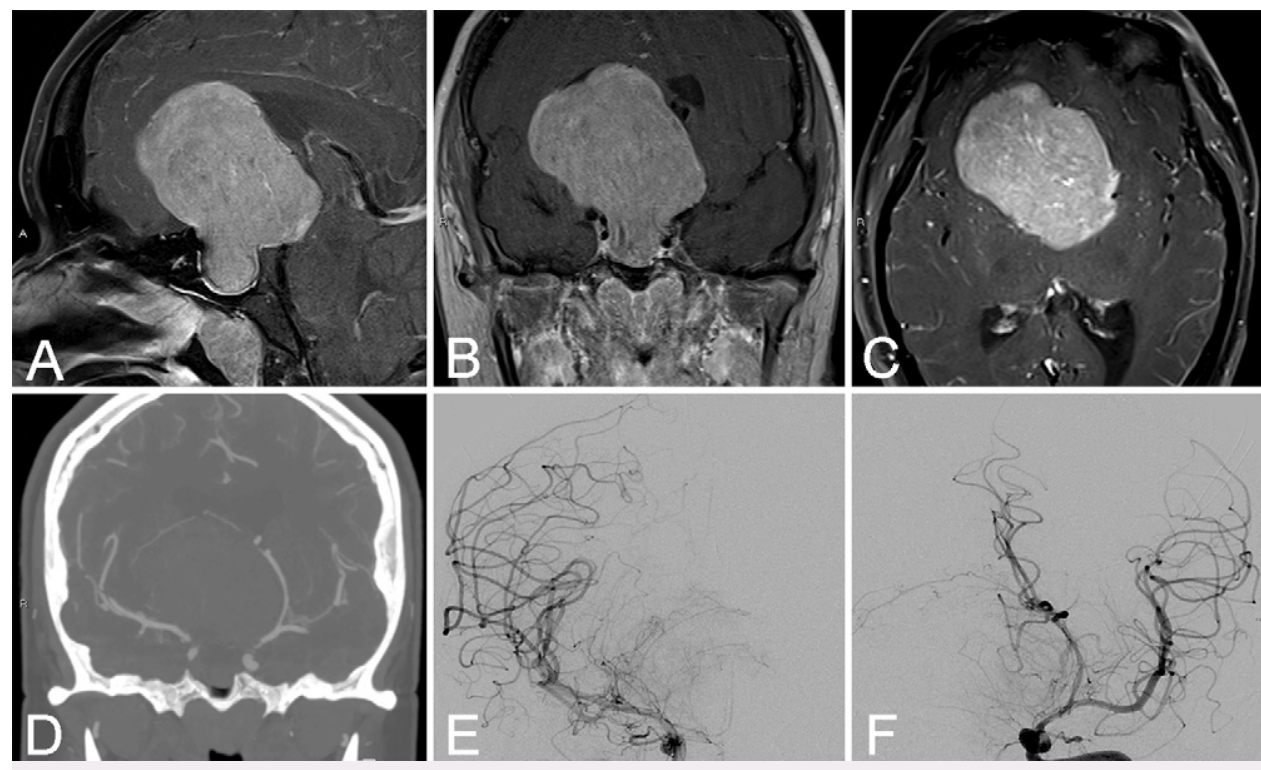

FIG. 1. A-C: Sagittal, coronal, and axial contrasted MRI showing giant pituitary macroadenoma with vascular proliferation. D: CTA showing lateral displacement of the left A1 segment and dropout of the right A1 segment with distal reconstitution. Digital subtraction angiography with anteroposterior views of the right ICA (E) and left ICA (F) injections showing vascular supply arising from multiple small branches originating from the bilateral ICA and MCA. Lateral view showed faint contrast extending to the anterior circulation (not shown). No suitable target was identified for preoperative endovascular embolization.

tomography angiography (CTA) showed the absence of the right $\mathrm{A} 1$ segment with distal reconstitution, as well as superior-lateral displacement of bilateral A2 segments (Fig. 1D). She underwent digital subtraction angiography for possible preoperative embolization. The tumor's vascular supply was from multiple small branches of the bilateral internal carotid arteries (ICAs) and middle cerebral arteries (MCAs) (Fig. 1E-F). No suitable endovascular targets were identified.

She underwent endonasal transsphenoidal resection in collaboration with an otorhinolaryngology specialist (S.C.P.). Seventy-degree reverse post scopes and retroflexed navigation suction instruments were used to maximize tumor visualization and extirpation. Intraoperative MRI demonstrated the extent of resection (Fig. 2). A craniotomy was not pursued.

Histopathological evaluation revealed loss of acinar architecture with sheets of pleomorphic cells possessing abundant acidophilic cytoplasm (Fig. 3A). Scattered cells were seen with cytoplasmic vacuoles and occasional mitotic figures (Fig. 3B). Immunohistochemical staining showed strong diffuse Pit-1 nuclear staining (Fig. 3C) and moderately elevated Ki-67 immunoreactivity (Fig. 3D). Fibrous bodies were stained for low-molecular-weight cytokeratin (CAM 5.2, Fig. 3E). Electron microscopy showed numerous dilated and giant mitochondria (Fig. 3F), confirming the diagnosis of acidophil stem cell adenoma.

MRI on postoperative day 1 showed small bilateral diffusion weighted imaging (DWI) abnormalities in the anterior cerebral artery (ACA) distribution (Fig. 4), from which she was asymptomatic. Postoperative CTA showed mild vasospasm of the left intracranial ICA and the left A1 segment. She was started on a steroid taper postoperatively. A detailed ophthalmological examination revealed no new

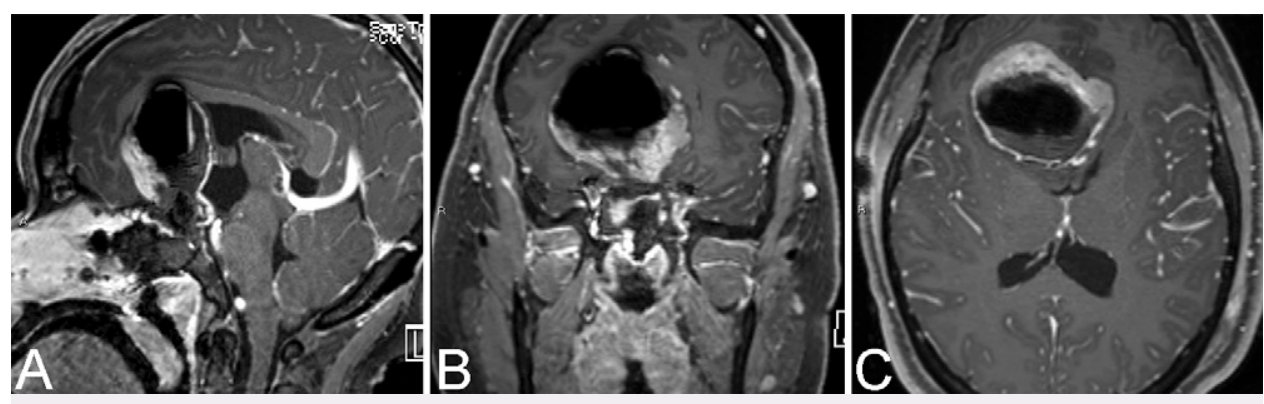

FIG. 2. A-C: Sagittal, coronal, and axial postoperative contrasted MRI studies showing residual tumor anteriorly. 


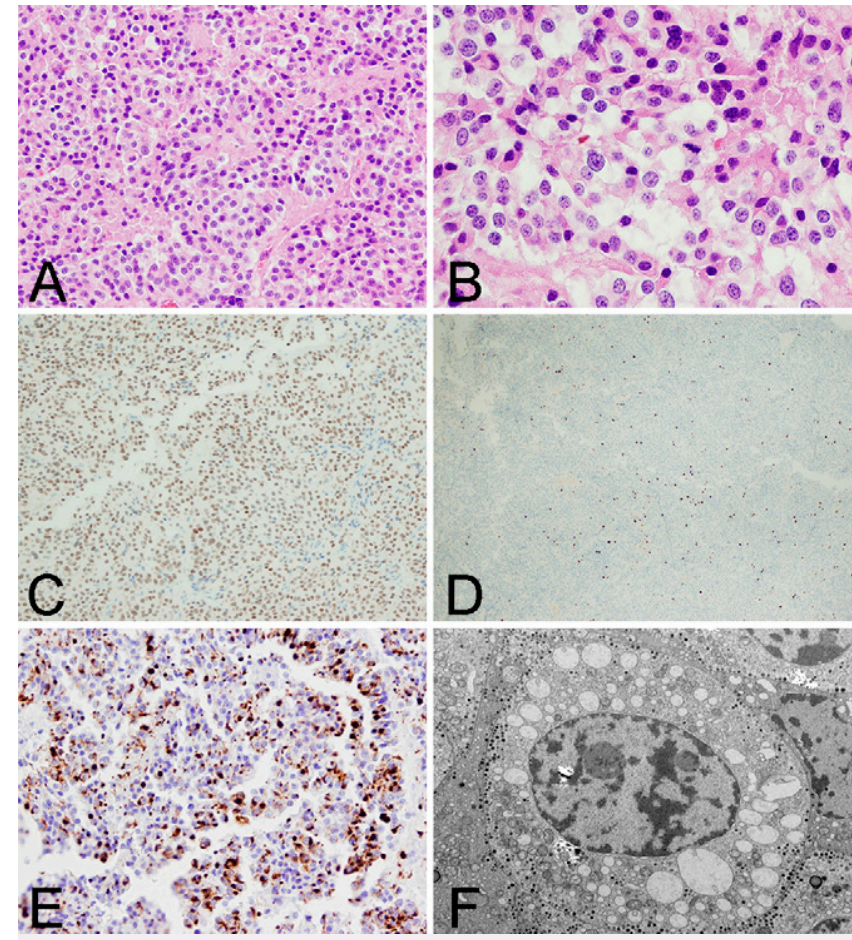

FIG. 3. Histopathological evaluation. A: Original magnification $\times 100$. Hematoxylin and eosin (H\&E) stain showing loss of acinar architecture with sheets of monomorphic cells. B: Original magnification $\times 400$. Higher power H\&E showing abundant acidophilic cytoplasm and scattered cells with cytoplasmic vacuoles and occasional mitotic figures. C: Original magnification $\times 100$. Pit-1 immunochemistry showing strong diffuse nuclear staining. D: Original magnification $\times 40$. Ki-67 immunohistochemical stain showing a moderately elevated proliferative index. E: Original magnification $\times 100$. CAM 5.2 immunohistochemical stain for low-molecular-weight cytokeratin. Globular cytoplasmic positivity is consistent with fibrous bodies. F: Original magnification $\times 8000$. Electron microscopy showing numerous dilated and giant mitochondria.

deficits but no immediate improvements in her vision. The remainder of her course was relatively unremarkable. She was discharged home on postoperative day 8 with plans for outpatient radiation therapy once the tumor cavity maximally consolidated.
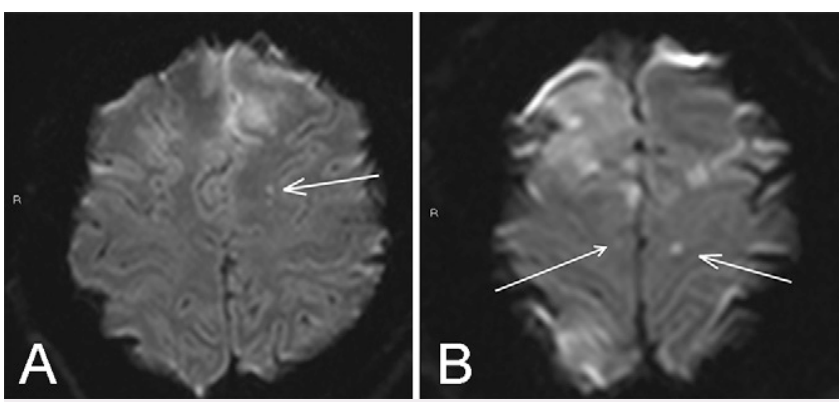

FIG. 4. A and B: Diffusion-weighted MRI on postoperative day 1 with small bilateral anterior cerebral artery distribution ischemic infarcts (arrows).

\section{Discussion}

Pituitary morphogenesis involves a complex interplay between several signaling molecules early in development. ${ }^{10}$ Selective expression of Pit-1 on mouse embryonic day e13.5-e17.5 gives rise to $\mathrm{Pit}^{+}{ }^{+}, \mathrm{GATA2}{ }^{-}$cells, which terminally differentiate to somatotrophs and lactotrophs expressing growth hormone and prolactin, respectively. ${ }^{11}$ Acidophil stem cell adenomas derive from residual undifferentiated Pit1 ${ }^{+}$precursors in adults. These rare tumors are defined by misplaced exocytosis, fibrous bodies, mitochondrial alterations, and oncocytic transformation..$^{12}$ They represent only about $4.3 \%$ of adenomas and display a more aggressive clinical profile with variable expression of growth hormone and prolactin. ${ }^{9}$

Given their aggressive growth pattern, acidophil stem cell adenomas typically come to medical attention early due to mass effect on adjacent structures. However, to our knowledge, no other reported cases have shown growth to the extent of forming a giant macroadenoma.

\section{Observations}

Despite its giant size and moderately elevated Ki-67 proliferative index, the tumor demonstrated limited invasiveness and appeared to remain within the tumor capsule. This is in contrast to other cancer stem cells that show more aggressive proliferation. ${ }^{13}$ This tumor retained the ability to recruit robust blood supply from bilateral ACA and MCA branches. Asymptomatic DWI changes are seen in up to $25 \%$ of patients after routine diagnostic cerebral angiography and are likely due to small silent emboli, especially given their higher frequency in patients with vascular risk factors ( $44 \%$ vs $13 \%, p=0.03) .{ }^{14}$ Our patient was similarly asymptomatic from her DWI changes.

A combined endonasal-transcranial approach was considered in this case. However, given the extent of endonasal resection achieved and the vascularity of the tumor, it was deemed safer to treat her residual tumor using radiation therapy. Her postoperative vision did not immediately improve. Patients with thin preoperative retinal nerve fiber layers measured by OCT often fail to improve at 6 weeks postoperatively, ${ }^{15}$ and this effect can persist up to 1 year. ${ }^{16}$ Earlier identification and treatment of patients with lesions abutting the chiasm may help decrease the risk of long-term deficits postoperatively.

\section{Lessons}

Here, we report the first known case of an acidophil pituitary stem cell adenoma presenting as a giant macroadenoma with profound visual deficits. We identify clinical and operative challenges unique to this rare tumor, as well as postoperative visual and functional status. Our study limitation is that this is a retrospective review of a single patient's data.

\section{References}

1. Ezzat S, Asa SL, Couldwell WT, et al. The prevalence of pituitary adenomas: a systematic review. Cancer. 2004;101(3):613-619.

2. Surawicz TS, McCarthy BJ, Kupelian V, et al. Descriptive epidemiology of primary brain and CNS tumors: results from the Central Brain Tumor Registry of the United States, 1990-1994. Neuro Oncol. 1999;1(1):14-25.

3. Jho HD, Carrau RL, Ko Y, Daly MA. Endoscopic pituitary surgery: an early experience. Surg Neurol. 1997;47(3):213-223.

4. Shrivastava RK, Arginteanu MS, King WA, Post KD. Giant prolactinomas: clinical management and long-term follow up. J Neurosurg. 2002;97(2):299-306.

5. Goel A, Nadkarni T, Muzumdar D, et al. Giant pituitary tumors: a study based on surgical treatment of 118 cases. Surg Neurol. 2004; 61(5):436-446. 
6. Müslüman AM, Cansever T, Yılmaz A, et al. Surgical results of large and giant pituitary adenomas with special consideration of ophthalmologic outcomes. World Neurosurg. 2011;76(1-2): 63-66, 141-148.

7. Matsuyama J, Kawase T, Yoshida K, et al. Management of large and giant pituitary adenomas with suprasellar extensions. Asian $\mathrm{J}$ Neurosurg. 2010;5(1):48-53.

8. Wang S, Lin S, Wei L, et al. Analysis of operative efficacy for giant pituitary adenoma. BMC Surg. 2014;14(1):59.

9. de Moraes DC, Vaisman M, Conceição FL, Ortiga-Carvalho TM. Pituitary development: a complex, temporal regulated process dependent on specific transcriptional factors. J Endocrinol. 2012:215(2):239-245.

10. Horvath E, Kovacs K, Singer W, et al. Acidophil stem cell adenoma of the human pituitary: clinicopathologic analysis of 15 cases. Cancer. 1981;47(4):761-771.

11. Dasen JS, O'Connell SM, Flynn SE, et al. Reciprocal interactions of Pit1 and GATA2 mediate signaling gradient-induced determination of pituitary cell types. Cell. 1999;97(5):587-598.

12. Horvath $\mathrm{E}$, Kovacs K, Singer W, et al. Acidophil stem cell adenoma of the human pituitary. Arch Pathol Lab Med. 1977;101(11): 594-599.

13. Prager BC, Bhargava S, Mahadev V, et al. Glioblastoma stem cells: driving resilience through chaos. Trends Cancer. 2020;6(3): 223-235.

14. Bendszus M, Koltzenburg M, Burger R, et al. Silent embolism in diagnostic cerebral angiography and neurointerventional procedures: a prospective study. Lancet. 1999;354(9190): 1594-1597.

15. Danesh-Meyer HV, Papchenko T, Savino PJ, et al. In vivo retinal nerve fiber layer thickness measured by optical coherence tomography predicts visual recovery after surgery for parachiasmal tumors. Invest Opthalmol Vis Sci. 2008;49(5): 1879-1885.

16. Park HH, Oh MC, Kim EH, et al. Use of optical coherence tomography to predict visual outcome in parachiasmal meningioma. J Neurosurg. 2015;123(6):1489-1499.

\section{Disclosures}

The authors report no conflict of interest concerning the materials or methods used in this study or the findings specified in this paper.

\section{Author Contributions}

Conception and design: Jane, Asuzu, Payne. Acquisition of data: Asuzu, Hakim, Payne. Analysis and interpretation of data: Asuzu, Hakim, Coss. Drafting the article: Asuzu, Hakim. Critically revising the article: Jane, Asuzu, Burke, Park. Reviewed submitted version of manuscript: Asuzu, Burke, Park, Payne. Created photomicrographs: Coss.

\section{Correspondence}

John A. Jane Jr.: University of Virginia, Charlottesville, VA. johnjanejr@virginia.edu. 